\title{
A Note on Detecting “More IFR-ness” Property of Life Distributions
}

\author{
Parameshwar V. Pandit ${ }^{1}$, Sujatha Inginashetty ${ }^{2}$ \\ ${ }^{1}$ Department of Statistics, Bangalore University, Bangalore, India \\ ${ }^{2}$ Department of Statistics, Gulbarga University, Gulbarga, India \\ Email: panditpv12@gmail.com, sujathasi_gug@rediffmail.com
}

Received August 25, 2012; revised September 28, 2012; accepted October 11, 2012

\begin{abstract}
In this paper, a problem of testing whether one life distribution possesses "more IFR" property than the other is considered. A new test procedure is proposed and the distribution of the test statistic is studied. The performance of the procedure is evaluated in terms of Pitman asymptotic relative efficiency. The consistency property of the test procedure is established. It is observed that the new procedure is better than the existing procedure in the literature.
\end{abstract}

Keywords: “More IFR” Property; U-Statistic; Pitman ARE

\section{Introduction}

A life is represented by a non-negative random variable $X$ with distribution function $F$ and survival function $\bar{F}=1-F$. Classes of life distributions based on notion of ageing have been introduced in the literature. One of the earliest and most important classes is the class of "Increasing Failure Rate" (IFR). We define IFR class below.

Definition 1.1. A distribution $F$ is said to be increasing failure rate (IFR), if $\frac{\bar{F}(x+t)}{\bar{F}(x)}$ is decreasing in $x$, for $t \geq$ 0.

Proschan and Pyke [1] proposed a test for testing exponentiality against IFR alternatives followed by Barlow and Proschan [2], Bickel and Doksum [3], Bickel [4] and many among others.

In practice, one might be interested in comparing two life distributions with respect to their possessing positive ageing property, particularly, IFR. Hollandar, Park and Proschan [5] developed a test procedure for testing the null hypothesis that two life distributions $F$ and $G$ are equal versus the alternative hypothesis that $F$ is more NBU than $G$. Tiwari and Zalkikar [6] proposed a test for testing the null hypothesis that two life distributions $F$ and $G$ are identical versus the alternative hypothesis that $F$ is "More increasing failure rate average" than $G$. Recently, Lim, Kim and Park [7] developed a class of test procedures for testing the null hypothesis that two life distributions $F$ and $G$ are equal against the alternative that $F$ is "more NBU at specified age" than $G$. However, the only test available for testing the null hypothesis that two life distributions $F$ and $G$ are identical against the alternative that $F$ is more IFR than $G$ is due to Pandit and Gudaganavar [8].

In this paper, we develop a simple test procedure for testing the null hypothesis that two life distributions $F$ and $G$ are equal against the alternative that $F$ is more IFR than $G$. The paper is organized as below: a test statistic is proposed for the problem of testing whether $F$ is more IFR than $G$ and its asymptotic distribution is established in Section 2. Section 3 contains the asymptotic relative efficiencies of the test proposed with the test due to Pandit and Gudaganavar [8] and some remarks and conclusions are presented in Section 4.

\section{The Proposed Two Sample "More IFR" Test}

Let $X_{1}, X_{2}, \cdots, X_{m}$ and $Y_{1}, Y_{2}, \cdots, Y_{n}$ denote two random samples from continuous life distributions $F$ and $G$ respectively. We want to develop test statistic for testing the null hypothesis.

$H_{0}: F=G$ (the common distribution is not specified);

Versus $H_{1}: F$ is "more IFR" than $G$ based on the two independent random samples.

Consider the parameter

$$
\gamma(F, G)=\gamma(F)-\gamma(G),
$$

where

$$
\gamma(F)=\int_{0}^{\infty} \int_{0}^{\infty} \bar{F}^{2}\left(\frac{x+t}{2}\right) \mathrm{d} F(x) \mathrm{d} F(t)
$$


and

$$
\gamma(G)=\int_{0}^{\infty} \int_{0}^{\infty} \bar{G}^{2}\left(\frac{x+t}{2}\right) \mathrm{d} G(x) \mathrm{d} G(t)
$$

Here $\gamma(F)$ and $\gamma(G)$ can be considered as the measure of degree of the IFR-ness. Mugadi and Ahmed [9] test used this measure as basis in the construction of test statistic for testing exponentiality against the IFR alternatives in one sample setting. If $F$ and $G$ belong to IFR class then $\gamma(F, G)$ can be taken as a measure for deciding whether $F$ is "more IFR" than $G$ or not. Under $H_{0}, \gamma(F, G)=0$ and it is strictly greater than zero under $H_{1}$. by

An unbiased estimator for $\gamma(F, G)$ which is given

$$
U_{m, n}=U_{1}-U_{2},
$$

where

$$
U_{1}=[m(m-1)(m-2)]^{-1} \sum_{i \neq} \sum_{j \neq} \sum_{k}\left[I\left(x_{i}\right)>\frac{\left(x_{j}+x_{k}\right)}{2}\right]
$$

and

$$
U_{2}=[n(n-1)(n-2)]^{-1} \sum_{i \neq} \sum_{j \neq} \sum_{k}\left[I\left(y_{i}\right)>\frac{\left(y_{j}+y_{k}\right)}{2}\right],
$$

where

$$
I(a>b)= \begin{cases}1 & \text { if } a>b \\ 0 & \text { otherwise }\end{cases}
$$

The asymptotic normality of the test $U_{m, n}$ is presented in the following theorem.

Theorem: As $n \rightarrow \infty, \sqrt{N}\left[U_{m, n}-\gamma(F, G)\right]$ is asymptotically normal with mean zero and variance

$$
\sigma^{2}=\frac{\sigma_{1}^{2}}{\lambda}+\frac{\sigma_{2}^{2}}{1-\lambda}
$$

where

$$
\begin{aligned}
\sigma_{1}^{2}= & 9 \operatorname{Var}\left(\int_{0}^{2 X_{1}} F\left(2 X_{1}-x\right) \mathrm{d} F(x)\right. \\
& \left.+2 \int_{0}^{\infty} \bar{F}\left(\frac{X_{1}+x}{2}\right) \mathrm{d} F(x)-\gamma(F)\right)
\end{aligned}
$$

and

$$
\begin{aligned}
\sigma_{2}^{2}= & 9 \operatorname{Var}\left(\int_{0}^{2 Y_{1}} G\left(2 Y_{1}-y\right) \mathrm{d} G(y)\right. \\
& \left.+2 \int_{0}^{\infty} \bar{G}\left(\frac{Y_{1}+y}{2}\right) \mathrm{d} G(y)-\gamma(G)\right)
\end{aligned}
$$

Under $H_{0}: \quad \sigma_{1}^{2}=\sigma_{2}^{2}=\sigma^{2}$ and is given by

$$
\sigma^{2}=\frac{\sigma_{1}^{2}}{\lambda(1-\lambda)} .
$$
theory that the limiting distribution of $\sqrt{N}\left[U_{m, n}-\gamma(F, G)\right]$ is asymptotic normal with mean 0 and variance $\sigma^{2}$, where $N=m+n$ is the combined sample size, $\sigma^{2}=\frac{\sigma_{1}^{2}}{\lambda}+\frac{\sigma_{2}^{2}}{1-\lambda}$ and $\lambda=\lim _{N \rightarrow \infty} \frac{m}{N}$ and it is assumed $0<\lambda<1$.

Here it is to be noted that the asymptotic mean of $\sqrt{N} U_{m, n}$ is zero, independent of unspecified common distribution $F_{0}$. However, the null asymptotic variance $\sigma^{2}=9 \xi_{1}\left(F_{0}\right) / \lambda(1-\lambda)$ does depend on $F_{0}$ through $\xi_{1}\left(F_{0}\right)$ and must be estimated from the data. To estimate $\sigma^{2}$, one possible way is to obtain consistent estimator for $\sigma^{2}$. For that,

Since

$$
\xi_{1}(F)=\operatorname{Var}\left[\psi_{1}\left(x_{1}\right)\right]
$$

where

$$
\psi_{1}\left(x_{1}\right)=E\left[\psi\left(X_{1}, X_{2}, X_{3}\right) \mid X_{1}=x_{1}\right]
$$

and

$$
\begin{aligned}
& \psi\left(X_{1}, X_{2}, X_{3}\right) \\
&=\frac{1}{3}\left[I\left(2 X_{1}>X_{2}+X_{3}\right)+I\left(2 X_{2}>X_{1}+X_{3}\right),\right. \\
&\left.+I\left(2 X_{3}>X_{1}+X_{2}\right)\right]
\end{aligned}
$$

we get

$$
\xi_{1}^{\prime}(F)=\frac{1}{m-1} \sum_{i=1}^{m}\left[\hat{\psi}_{1}\left(x_{i}\right)-U_{1}\right]^{2}
$$

where

$$
\hat{\psi}_{1}\left(x_{i}\right)=\frac{1}{m-1} \sum_{\substack{i=1 \\ j \neq k \neq i}}^{m} \psi\left(x_{i}, x_{j}, x_{k}\right)
$$

In similar fashion, we define

$$
\xi_{1}^{\prime}(G)=\frac{1}{(n-1)} \sum_{j=1}^{n}\left[\hat{\psi}_{1}\left(y_{j}\right)-U_{2}\right]
$$

where

$$
\hat{\psi}_{1}\left(y_{i}\right)=\frac{1}{n-1} \sum_{\substack{i=1 \\ j \neq k \neq i}}^{m} \psi\left(y_{i}, y_{j}, y_{k}\right)
$$

Then it is easily verified that

$$
U_{1}=\frac{1}{m} \sum_{i=1}^{m} \hat{\psi}_{1}\left(x_{i}\right) \text { and } U_{2}=\frac{1}{n} \sum_{j=1}^{n} \hat{\psi}_{1}\left(y_{j}\right) .
$$

$\xi_{1}^{\prime}(F)$ and $\xi_{1}^{\prime}(G)$ are consistent estimators of $\xi_{1}(F)$ and $\xi_{1}(G)$, and this consistent estimator $\hat{\sigma}_{N}^{2}$ is obtained by replacing $\xi_{1}(F)$ and $\xi_{1}(G)$ by $\xi_{1}^{\prime}(F)$ and $\xi_{1}^{\prime}(G)$, respectively in the expression of $\sigma^{2}$. Hence the estimator for $\sigma_{N}^{2}$ is obtained as 


$$
\hat{\sigma}_{N}^{2}=\left(9 N \xi_{1}^{\prime}(F) / m\right)+\left(9 N \xi_{1}^{\prime}(G) / n\right) .
$$

By the consistency of $\hat{\sigma}_{N}^{2}$ and Slutsky's theorem $\sqrt{N} V \hat{\sigma}_{N}^{-1}$ is asymptotically $N(0,1)$ under $H_{0}$.

The approximate $\alpha$-level test of $H_{0}$ versus $H_{1}$ rejects $H_{0}$ in favour of $H_{1}$ if

$$
\sqrt{N} U_{m, n} /\left\{\left(9 N \xi_{1}^{\prime}(F) / m\right)+\left(9 N \xi_{1}^{\prime}(G) / n\right)\right\}^{1 / 2}>z_{\alpha}
$$

where $z_{\alpha}$ is the upper $\alpha$-percentile point of the normal distribution. This ensures the consistency of the two sample IFR test against the class of $(F, G)$ pairs satisfying $\gamma(F)-\gamma(G)>0$.

\section{Asymptotic Relative Efficiency}

We study the asymptotic efficacy of $U_{m, n}$, for two pairs of distribution $\left(F_{i, \theta}, G\right)$. Here, we assume that $G$ is an exponential distribution with mean one. The different distributions considered here for $\left(F_{i, \theta}\right)$ are given below:

1) Linear Failure Rate Distribution

$$
\bar{F}_{2 . \theta}(x)=\exp \left\{-\left(x+\theta \frac{x^{2}}{2}\right)\right\}, x>0, \theta \geq 0
$$

2) Makeham Distribution

$$
\bar{F}_{3 . \theta}(x)=\exp \left\{-\left[x+\theta\left(x+\mathrm{e}^{-x}-1\right)\right]\right\}, \theta \geq 0, x \geq 0
$$

The Pitman asymptotic efficacy is given by

$$
\operatorname{Eff}\left(U_{m, n}, F_{i . \theta}, G\right)=\sigma_{0}^{-2}(v)\left\{\frac{\mathrm{d}}{\mathrm{d} \theta} \gamma\left(F_{i, \theta}, G\right)\right\}_{\theta \rightarrow \theta_{0}}^{2} .
$$

The mean of the proposed test is

$$
\begin{aligned}
\gamma\left(F_{i, \theta}, G\right)= & \int_{0}^{\infty} \int_{0}^{\infty} \bar{F}\left(\frac{x+y}{2}\right) \mathrm{d} F(x) \mathrm{d} F(y) \\
& -\int_{0}^{\infty} \int_{0}^{\infty} \bar{G}\left(\frac{x+y}{2}\right) \mathrm{d} G(x) \mathrm{d} G(y)
\end{aligned}
$$

since $G$ is exponential, $\gamma(G)=\frac{4}{9}$.

The asymptotic efficacies of the proposed test for Linear Failure Rate distribution and Makeham

distribution are $\frac{0.8681}{\lambda(1-\lambda)}$ and $\frac{1.4062}{\lambda(1-\lambda)}$ respectively.

Next, we compute ARE of the two sample test based on $U_{m, n}$ proposed here, by considering a sequence of alternatives $\left(F_{\theta \phi}, G_{\theta}\right)$ where $F_{\theta \phi}$ is LFR or Makeham distribution with parameter $\theta \phi$, where $\phi=1+\frac{a}{\sqrt{N}}$, “ $a$ " being arbitrary positive constant, and $G_{\theta}$ is LFR or Makeham with parameter $\theta$. It is to be noted that as
$N \rightarrow \infty$, the sequence of alternatives converges to the null hypothesis. That is $G_{\theta}=F_{\theta \phi}$ when $\phi=1$. The efficacy of the $V$ test is given by

$$
\operatorname{eff}\left(U_{m, n}\right)=\frac{\left\{\gamma^{\prime}(F, G)\right\}_{\phi=1}}{\sigma_{H_{0}}\left(U_{m, n}\right)},
$$

where $\sigma_{H 0}\left(U_{m, n}\right)$ is null asymptotic standard deviation of $U_{m, n}$, and

$$
\frac{\gamma^{\prime}(F, G)}{\phi=1}=\left[\frac{\mathrm{d}}{\mathrm{d} \phi} \gamma(F, G)\right]_{\phi=1} .
$$

The AREs of $U_{m, n}$ relative to $V$, the test due to Pandit and Gudaganavar [8] for different values of $\theta$ are presented in Table 1.

\section{Some Remarks}

1) A simple test procedure for testing the null hypothesis that two life time distributions are identical against the alternative that one possesses more IFR property than another. The test proposed is based on the measure considered by Mugadi and Ahmad [9] for one sample problem.

2) The only test available for the above stated problem in the literature is due to Pandit and Gudaganavar [8]. Hence, only Pitman AREs relative to the test due to Pandit and Gudaganavar [8] are computed for the few alternatives by specifying the common null distribution to be exponential. However, similar testing problems with more NBU/IFRA/NBU- $t_{0}$ property are considered by few authors, namely, Hollander, Park and Proschan [5], Tiwari and Zalkikar [6] and Lim, Kim and Park [7].

3) We have also computed Pitman AREs relative to Pandit and Gudaganavar [8] for those alternatives with common null distributions being Makeham and Linear failure rate distributions.

4) It is observed that the new test proposed here is better than Pandit and Gudaganavar [8] test for the distributions considered in this paper. However, the performance of Pandit and Gudaganavar [8] test is better for Weibull distribution.

\section{Acknowledgements}

Authors thank the Editorial Board and reviewers for their

Table 1. AREs of $U_{m, n}$ relative to $V$.

\begin{tabular}{ccc}
\hline$\theta$ & LFR & Makeham \\
\hline 2 & 1.7814 & 1.5386 \\
3 & 1.9358 & 1.4612 \\
4 & 1.9483 & 1.4475 \\
5 & 1.9822 & 1.4493 \\
\hline
\end{tabular}


valuable comments which lead to this revised version of the paper.

\section{REFERENCES}

[1] F. Proschan and R. Pyke, "Tests for Monotone Failure Rate," Proceedings of the Fifth Berkeley Symposium on Mathematical Statistics and Probability, Vol. 3, 1967, pp. 293-312.

[2] R. E. Barlow and F. Proschan, "A Note on Tests of Monotone Failure Rate Based on Incomplete Data," The Annals of Mathematical Statistics, Vol. 40, No. 2, 1969, pp. 595-600. doi:10.1214/aoms/11776927

[3] P. J. Bickel and K. Doksum, “Test for Monotone Failure Rate Based on Normalized Spacings," The Annals of Mathematical Statistics, Vol. 40, No. 4, 1969, pp. 12161235. doi:10.1214/aoms/1177697498

[4] P. J. Bickel, “Tests of Monotone Failure Rate II,” The Annals of Mathematical Statistics, Vol. 40, No. 4, 1969, pp. 1250-1260. doi:10.1214/aoms/1177697498
[5] R. M. Hollander, D. H. Park and F. Proschan, "Testing Whether F Is 'More NBU' than Is G,' Microelectronics Reliability, Vol. 26, No. 1, 1986, pp. 39-44. doi:10.1016/0026-2714(86)90769-9

[6] R. C. Tiwari and J. N. Zalkikar, "Testing Whether F Is 'More IFRA than G',' Microelectronics Reliability, Vol. 28, No. 5, 1988, pp. 703-712. doi:10.1016/0026-2714(88)90006-6

[7] J. H. Lim, D. K. Kim and D. H. Park, "Tests for Detecting More NBU-ness at Specific Age,” Australian and New Zealand Journal of Statistics, Vol. 47, No. 3, 2005, pp. 329-337.

[8] P. V. Pandit and N. V. Gudaganavar, "On Two-Sample Test for Detecting Differences in the IFR Property of Life Distributions," African Journal of Mathematical and Computer Science Research, Vol. 2, No. 2, 2009, pp. 25-29.

[9] A. R. Mugadi and I. A. Ahmad, "Classes of Statistics for Testing Exponentiality versus IFR or NBUE Alternatives," Calcutta Statistical Association Bulletin, Vol. 62, No. 245246, 2010, pp. 17-29. 